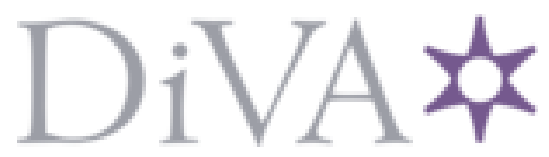

http://www.diva-portal.org

This is the published version of a paper published in Physical Review A. Atomic, Molecular, and Optical Physics.

Citation for the original published paper (version of record):

Dutta, O., Jääskeläinen, M., Meystre, P. (2005)

Polarizing beam splitter for dipolar molecules.

Physical Review A. Atomic, Molecular, and Optical Physics, 71(5): 051601

http://dx.doi.org/10.1103/PhysRevA.71.051601

Access to the published version may require subscription.

N.B. When citing this work, cite the original published paper.

Permanent link to this version:

http://urn.kb.se/resolve?urn=urn:nbn:se:du- 11210 


\title{
Polarizing beam splitter for dipolar molecules
}

\author{
O. Dutta, M. Jääskeläinen, ${ }^{*}$ and P. Meystre \\ Optical Sciences Center and Department of Physics, The University of Arizona, Tucson, Arizona 85721, USA
}

(Received 22 December 2004; published 6 May 2005)

\begin{abstract}
We propose a coherent beam splitter for polarized heteronuclear molecules based on a stimulated Raman adiabatic passage scheme that uses a tripod linkage of electrotranslational molecular states. We show that for strongly polarized molecules the rotational dynamics imposes significantly larger Rabi frequencies than would otherwise be expected, but within this limitation, a full transfer of the molecules to two counterpropagating ground-state wave packets is possible.
\end{abstract}

DOI: 10.1103/PhysRevA.71.051601

PACS number(s): 03.75.Be, 42.50.-p, 33.80.-b, 03.65.-w

The rapidly growing field of coherent matter-wave optics [1] has recently been extended to quantum-degenerate fermionic atoms [2] and to ultracold alkali dimers [3], opening up the way to the nonlinear optics of fermions and molecules and to superchemistry [4]. So far, two methods have led to the creation of ultracold atomic dimers: photoassociation [5], which results in the production of ground-state molecules; and Feshbach resonances, which yield weakly bound dimers. An exciting recent development is the observation of Feshbach resonances between different atomic species [6,7], which hints at the possibility of creating quantum-degenerate samples of heteronuclear dimers in the near future. Such ultracold dipolar molecules (see [8] for recent developments) hold much promise for applications from quantum information [9] to fundamental studies of dipolar superfluids [10]. In addition, their potential for atom optics applications appears considerable, motivated to a large extent by the ability to trap and guide them with modest electric field gradients. In this context, the recent demonstration of a large, centimeter-sized storage ring [11] hints at the potential for orders-ofmagnitude improvements in neutral-particle-based inertial sensors.

An essential step in the application of such devices to guided matter-wave interferometry is the capability to coherently split an ultracold molecular sample into two counterpropagating beams. Ideally, this should be achieved in the presence of the static electric fields that provide the waveguiding elements and help maintain control over the inherent two-body dipole-dipole interaction by polarizing the molecular beam. However, this alignment is achieved at the cost of exciting a number of rotational energy levels, thereby complicating any beam-splitting mechanism. Hence, understanding the coherent splitting of a molecular cloud in the presence of polarizing electric fields is a key step toward molecular optics. This paper analyzes a polarizing beam splitter that combines a (quasi) static electric field with a sequence of laser pulses that split the molecules into two counterpropagating wave packets, while simultaneously transferring them from the weakly bound state in which they are initially formed via Feshbach resonances to their electrovibrational ground state.

*Corresponding author. Email address: mrq@optics.arizona.edu
We consider a stimulated Raman adiabatic passage (STIRAP) scheme that relies on the usual "counterintuitive" laser pulse sequence [12]. Atomic mirrors and beam splitters based on this technique were proposed in Ref. [13], which demonstrated how the application of STIRAP to multilevel atomic systems can generate coherent superpositions of atoms in two ground-state sublevels of opposite center-of-mass momenta. A further extension allowing controlled splitting was theoretically investigated and experimentally implemented in [14]. Reference [15] generalized that scheme to produce a superposition of internal states with two momentum components in each. Our goal is to extend these ideas to the realization of a large-angle beam splitter that produces a coherent superposition of polarized counterpropagating molecular wave packets in a single electrovibrational ground state, an important requirement for applications such as ring interferometers where interference can then be observed without the application of a second STIRAP sequence. We present the basic principle of the beam splitter in a simple case and later discuss the impact of the rotational degrees of freedom. For concreteness, we consider the specific example of the RbCs dimer, for which recent theoretical spectroscopic data are available [16]. The key elements of the scheme are sketched in Fig. 1, which shows the potential curves of the $\mathrm{RbCs}$ dimer and the transitions involved in the STIRAP process (a), as well as the laser geometry relative to the molecular cloud (b). STIRAP achieves the transfer of the molecules from the weakly bound initial state ${ }^{3} \Sigma^{+}$to the molecular ground state ${ }^{1} \Sigma^{+}$via a counterintuitive sequence of Stokes and anti-Stokes pulses. The feasibility of transferring ultracold heteronuclear molecules produced by Feshbach resonances has been investigated in [17] for a number of different dimers including RbCs. The matching of the turning points in these molecules implies large transition moment and Franck-Condon factors for both the initial and the final step of a stimulated Raman transition. In particular, the transfer to the $X^{1} \Sigma^{+}$state of RbCs used is pursued in RbCs photoassociation experiments (see Ref. [18]).

Due to momentum conservation, the absorption of a linear superposition of photons with opposite momenta results in a final electronic state in a coherent superposition of two counterpropagating wave packets. We are interested in realizing a situation where the final momentum of the particles along $\mathbf{k}_{0}$, the transverse direction, is equal to zero, $k_{\perp}=\left|k_{0}\right|+\left|k_{ \pm}\right| \cos \phi$ $=0$, resulting in counterpropagating atomic wave packets. 


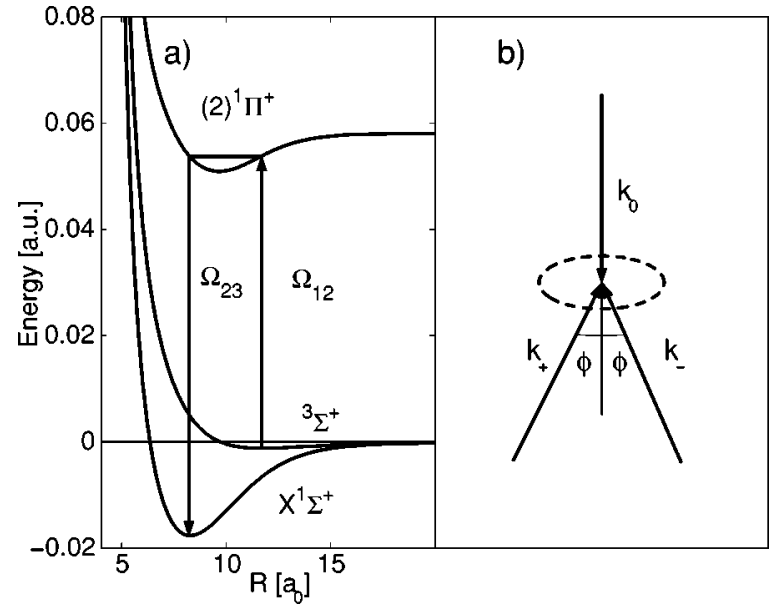

FIG. 1. (a) Electronic transitions used in the STIRAP process, for the case of RbCs. The molecular energy curves are taken from Ref. [16]. (b) The laser geometry, with the Stokes fields labeled $k_{ \pm}$ and the anti-Stokes field $k_{0}$.

Combined with the conservation of energy condition $\left(\hbar^{2} / 2 M\right)\left(\left|k_{ \pm}\right|^{2}-\left|k_{0}\right|^{2}\right)=\Delta E$, where $\Delta E$ is the energy difference between the initial $\left({ }^{3} \Sigma^{+}\right)$and final $\left(X^{1} \Sigma^{+}\right)$electronic states, and $M$ is the mass of the molecules. For $\left|k_{+}\right|=\left|k_{-}\right|$this gives for the angle $\phi$

$$
\cos \phi=\sqrt{1-(2 M \Delta E) /\left(\hbar^{2}\left|k_{ \pm}\right|^{2}\right)} .
$$

For the case of $\mathrm{RbCs}$, we have $2 M \Delta E /\left(\hbar^{2}\left|k_{ \pm}\right|^{2}\right) \approx 0.5$ so that $\phi \approx 30^{\circ}$, and consequently the momentum of the final states is $k_{R}=\left|k_{ \pm}\right| \sin \phi \approx 0.5 k_{ \pm}$.

Restricting ourselves from now on to the one-dimensional situation of zero transverse momentum, the STIRAP Hamiltonian for the three-level configuration of Fig. 1 is given by

$$
\hat{H}_{\text {STIRAP }}=\left[\begin{array}{ccc}
0 & \Omega_{12}(t) & 0 \\
\Omega_{12}(t) & 0 & \Omega_{23}(t) \cos \left(k_{R} z\right) \\
0 & \Omega_{23}(t) \cos \left(k_{R} z\right) & 0
\end{array}\right],
$$

where $\Omega_{i j}(t)$ is the Rabi frequency that describes the strength of the electric dipole coupling between levels $|i\rangle$ and $|j\rangle$. In the STIRAP configuration, the pulse $\Omega_{23}(t)$ precedes $\Omega_{12}(t)$, and the population of the intermediate state $(2)^{1} \Pi^{+}$remains negligible. The effective Rabi frequency of the ${ }^{3} \Sigma^{+} \rightarrow X^{1} \Sigma^{+}$ transition is then $\Omega(t) \equiv \sqrt{\Omega_{12}(t)^{2}+\Omega_{23}(t)^{2}}$. It is known that the transfer from initial to final state is robust against small changes in the pulse parameters provided that the condition $\Omega \tau \gg 1$ is fulfilled, where $\Omega$ is the peak value of $\Omega(t)$.

Figure 2 shows the result of a typical numerical simulation for Gaussian-shaped pulses of $1 \mathrm{~ns}$ duration and an initial molecular wave packet at rest, $k_{z}=0$, carried out by standard numerical techniques [19]. We note that when the spatial extent $\Delta z$ of the initial wave packet is large compared with the recoil momentum, $k_{R} \Delta z \ll 1$, then the momentum states before and after the adiabatic transfer are almost orthogonal. The problem at hand can then be thought of as a version of STIRAP that uses a tripod linkage of electrotranslational molecular states, the counterpropagating final wave
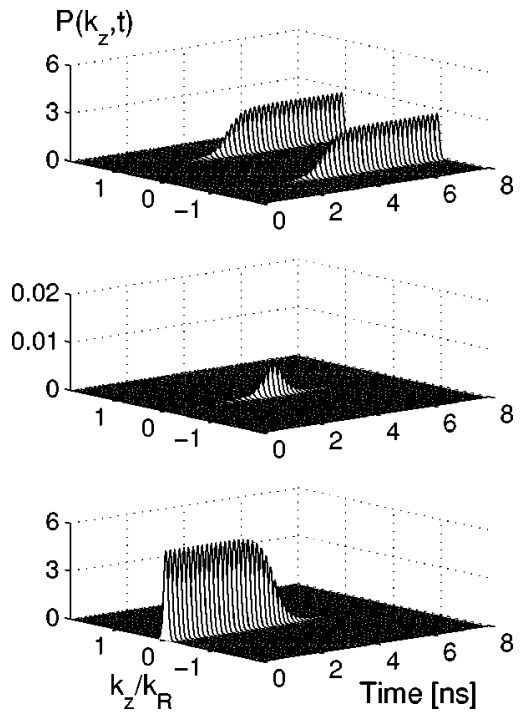

FIG. 2. Momentum space probability densities of the three electronic states of interest, ${ }^{3} \Sigma^{+}$(bottom curve), (2) ${ }^{1} \Pi^{+}$(middle curve), and $X^{1} \Sigma^{+}$(top curve) as a function of time. The momentum is in units of the recoil momentum $k_{R}$. In this example, the Rabi frequencies $\Omega_{12}(t)$ and $\Omega_{23}(t)$ are Gaussians of width equal to $1 \mathrm{~ns}$, peak value $40 \times 10^{9} \mathrm{rad} \mathrm{s}^{-1}$, and $\Omega_{23}$ precedes $\Omega_{12}$ by $2 \mathrm{~ns}$.

packets corresponding to distinct, quasiorthogonal states. This suggests treating the momentum states as quasimodes [20], with the inclusion of $k_{z}=0$ for the initial and intermediate states, and $k_{z}= \pm k_{R}$ for the final electronic state, a procedure that greatly simplifies the numerical treatment.

The three curves of Fig. 2 show the evolution of the momentum space probability densities of the three electronic states of interest: ${ }^{3} \Sigma^{+}$(bottom curve), $(2)^{1} \Pi^{+}$(middle curve), and $X^{1} \Sigma^{+}$(top curve). The transfer of the initial wave packet to two counterpropagating ground-state wave packets without any significant population of the intermediate state is readily apparent (note the different vertical scales for the intermediate state population) and as expected, the probability densities for the two resulting counterpropagating wave packets are equal. We have verified numerically that the process has the familiar robustness of STIRAP.

We now turn to a discussion of the influence of the static electric fields used in the guiding and the alignment of the molecules. The fact that heteronuclear molecules have a permanent dipole moment makes it crucial to control their orientational distribution, since this determines their interaction energy in a static external field. For the purpose of trapping polar molecules in a static electric field, that distribution has to be aligned. This can be achieved through "brute-force" alignment by ramping up a quasistatic electric field [21,22] over a time scale long compared with the rotation period of the molecules. This results in a coherent superposition of angular momentum states that interfere to produce a probability distribution aligned in space [23].

The wave-packet treatment considered so far can be thought of as having considered a single rotational final state. The full transfer process must account for a manifold of interfering STIRAP transitions characterized in general by distinct detunings, the result of the slightly differing Clebsch- 

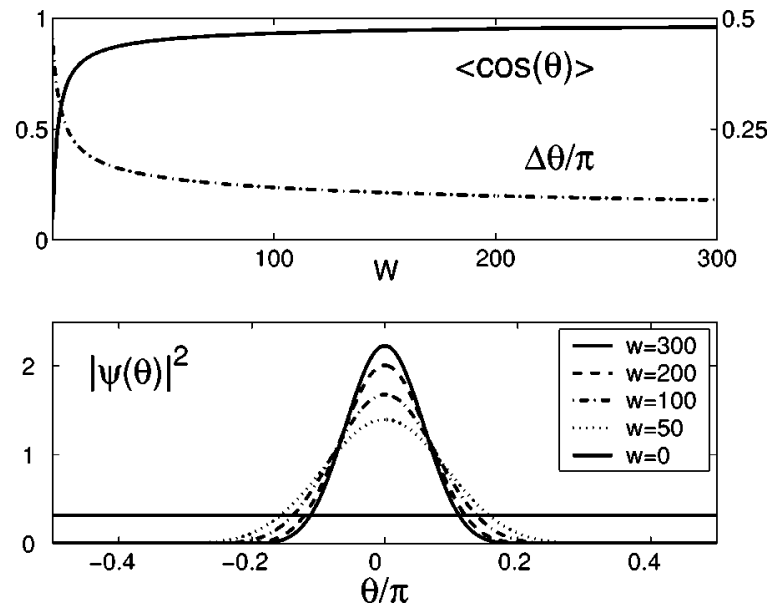

FIG. 3. Top: $\langle\cos (\theta)\rangle$ (solid line) and $\Delta \theta=\sqrt{\left\langle\theta^{2}\right\rangle-\langle\theta\rangle^{2}}$ (dashdotted line) as a function of $w$. Bottom: The angular probability distribution $|\Psi(\theta)|^{2}$ for several values of $w$.

Gordan coefficients between individual rotational states. The rotational and orientational parts of the molecular Hamiltonian are given by

$$
\hat{V}=B_{e} \hat{J}^{2}+\mathbf{d} \cdot \mathbf{E}=B_{e} \hat{J}^{2}+d E \cos \theta,
$$

where $B_{e}$ is the rotational constant, $d$ is the magnitude of the static dipole moment $\mathbf{d}$, and $E$ the magnitude of the (quasistatic) trapping and guiding electric field $\mathbf{E}$, taken to be transverse to the direction of propagation and forming an angle $\theta$ with the molecular dipole moment.

We proceed by expanding the molecular state vector in a basis of momentum quasimodes and angular momenta as $|\Psi(t)\rangle=\Sigma_{i, k_{i},} c_{J, k_{i}}^{i}(t)\left|i, k_{i}, J\right\rangle$, where $i$ labels the electronic state, $k_{i}$ the momentum quasimode, and $J$ the total angular momentum of the molecule [24]. We assume that the Feshbach resonance produces molecules in the ground state $J$ $=0$ of the rotational Hamiltonian $\hat{V}$ at zero field $E=0$. The guiding electric field is then turned on adiabatically after creation of the weakly bound molecules, but before switching on the STIRAP laser pulses. The rotational state of the molecules prior to the adiabatic transfer is therefore taken to be the ground state of the Hamiltonian (2). The angular distribution of that state depends only on the dimensionless ratio $w \equiv d E / B_{e}$ between the orientational and rotational energies [22]. This is illustrated in Fig. 3, which shows the expectation value $\langle\cos \theta\rangle$ and $|\Psi(\theta)|^{2}$, the probability to find the molecule in a state tilted by $\theta$ with respect to the electric field, for several values of $w$. Strongly aligned distributions require electric fields of the order of $10-70 \mathrm{kV} / \mathrm{cm}$ for the heteronuclear dimers under consideration.

A strongly aligned state consists of many rotational states, complicating the STIRAP by introducing a number of competing molecular states. In particular, the rotational part of $\hat{V}$ has diagonal elements of the form $B_{e} J(J+1)$ that introduce detunings that significantly impact the efficiency of the STIRAP transfer. This is illustrated in Fig. 4, which summarizes the results of a multilevel numerical analysis that fully accounts for the dynamics associated with the rotational con-

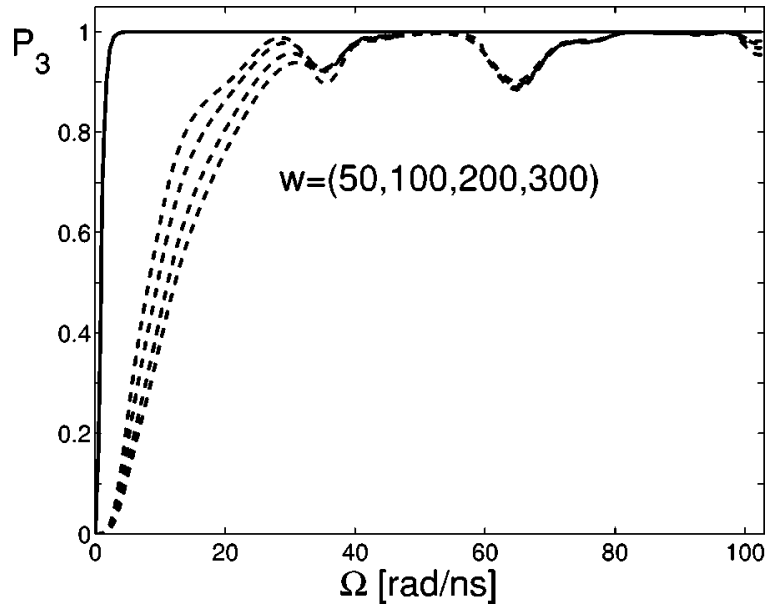

FIG. 4. Transfer probability $P_{3}$ vs Rabi frequency $\Omega$ for several values of the parameter $w$, with curves corresponding to increasing $w$ from left to right. The solid line shows the transfer probability for the case $B_{e}=0$ for reference. Pulse length $\tau=1 \mathrm{~ns}$.

tribution to the molecular Hamiltonian. It shows the total transfer probability $P_{3}=\lim _{t \rightarrow \infty} \int_{-\pi / 2}^{\pi / 2} d \theta\left|\Psi_{3}(\theta, t)\right|^{2}$ where

$$
\Psi_{3}(\theta, t)=\sum_{J, k_{3}} c_{J, k_{3}}^{3}(t) \sqrt{\frac{2 J+1}{8 \pi^{2}}} P_{J}(\cos \theta),
$$

and $P_{J}(\cos \theta)$ are the associated Legendre polynomials, as a function of the Rabi frequency $\Omega$ for several values of $w$. The most obvious consequence of the rotational dynamics is that high transfer probabilities to the final counterpropagating wave packets now require much higher Rabi frequencies. Figure 4 shows that for strongly polarized molecular fields, this increase can be as much as an order of magnitude or more. Such an increase is intuitively expected in order to suppress the effect of the detunings of the individual rotational states. To achieve full transfer requires the coupling Rabi frequency to be significantly larger than the rotational energy, $B_{e}\left\langle\hat{J}^{2}\right\rangle \ll \Omega$. Typical values of $\left\langle\hat{J}^{2}\right\rangle$ for the electric field are in the range $4-12$, and $B_{e}=3 \times 10^{9} \mathrm{rad} \mathrm{s}^{-1}$, giving Rabi frequencies of order $\Omega=10^{10} \mathrm{rad} \mathrm{s}^{-1}$, and corresponding laser intensities $I=10^{7}-10^{8} \mathrm{~W} \mathrm{~m}^{-2}$. The positions of the pronounced dips in $P_{3}$ were found numerically to depend on the value of the rotational constant $B_{e}$, and result from interferences between the contributions of various rotational levels. Except for these features, the inclusion of the rotational dynamics, $B_{e}>0$, does not significantly affect the alignment of the final state, only the transfer efficiency. This indicates that the STIRAP transfer is dominated by transitions that do not change the rotational quantum number $J$ between the initial and final electronic states. Finally, we note that relaxing the condition that the Feshbach resonance produces molecules in $J=0$ leads to the excitation of a larger number of rotational states when applying the static field, and the need to increase $\Omega$ by an additional factor of two or so.

In summary, we have proposed a coherent beam splitter for polarized heteronuclear molecules using a tripod linkage of electrotranslational molecular states. The rotational dynamics imposes Rabi frequencies larger by about an order of 
magnitude than would otherwise be expected, but within this limitation, a full transfer of the molecules to two counterpropagating ground-state wave packets is possible. This offers the possibility of extending matter-wave interferometry to the promising domain of ultracold, polar molecules. This scheme is easily extended to include several momentum states in order to split molecular wave packets into more exotic combinations by the application of several Stokes pulses. The role of collisions and other many-body effects in the efficiency of creation of molecules using STIRAP has been discussed earlier $[25,26]$. We note that since $s$-wave scattering is suppressed for the case of fermionic dimers such as $\mathrm{RbCs}$, dipole-dipole interactions will be dominant. For typical densities they correspond to interaction energies of the order of $10^{4} \mathrm{rad} \mathrm{s}^{-1}$ [27], a value small compared to the Rabi frequencies and hence of negligible impact on the transfer efficiency. The effect of quantum statistics on the transfer is left for future investigations.

This work is supported in part by the U.S. Office of Naval Research, by the National Science Foundation, by the U.S. Army Research Office, by the National Aeronautics and Space Administration, and by the Joint Services Optics Program.
[1] P. Meystre, Atom Optics (Springer, New York, 2001).

[2] G. Roati et al., Phys. Rev. Lett. 94, 040405 (2005).

[3] J. R. Abo-shaer et al., Phys. Rev. Lett. 92, 230402 (2004).

[4] D. J. Heinzen, R. Wynar, P. D. Drummond, and K. V. Kheruntsyan, Phys. Rev. Lett. 84, 5029 (2000).

[5] A. J. Kerman et al., Phys. Rev. Lett. 92, 033004 (2004); 92, 153001 (2004).

[6] S. Inouye et al., Phys. Rev. Lett. 93, 183201 (2004).

[7] C. A. Stan et al., Phys. Rev. Lett. 93, 143001 (2004).

[8] Eur. Phys. J. D 31, 149 (2004), topical issue on ultracold dipolar molecules, edited by J.Doyle, B.Friedrich, R.V.Krems, and F.Masnou-Seeuws.

[9] G. K. Brennen et al., Phys. Rev. Lett. 82, 1060 (1999).

[10] B. Damski et al., Phys. Rev. Lett. 90, 110401 (2003); 90, 1060 (1999); G. K. Brennen et al., Phys. Rev. A 65, 022313 (2002); D. DeMille, Phys. Rev. Lett. 88, 067901 (2002).

[11] F. M. H. Crompvoets et al., Nature (London) 411, 6834 (2001).

[12] K. Bergmann, H. Theuer, and B. W. Shore, Rev. Mod. Phys. 70, 1003 (1998).

[13] P. Marte, P. Zoller, and J. Hall, Phys. Rev. A 44, R4118 (1991)

[14] H. Theuer, et al., Opt. Express 4, 77 (1999).
[15] L. I. Plimak, Y. V. Rozhdestvensky, M. K. Olsen, and M. J. Collett, Phys. Rev. A 63, 023608 (2001).

[16] A. R. Allouche et al., J. Phys. B 33, 2307 (2000).

[17] W. C. Stwalley, Eur. Phys. J. D 31, 221 (2004).

[18] T. Bergeman et al., Eur. Phys. J. D 31 (2004).

[19] B. M. Garraway and K.-A. Suominen, Rep. Prog. Phys. 58, 365 (1995); N. Balakrishnan, C. Kalyaraman, and N. Sathyamurthy, Phys. Rep. 280, 80 (1997); J. Z. H. Zhang, Theory and Application of Quantum Molecular Dynamics (World Scientific, Singapore, 1999); J. Yeazell and U. Turgay, The Physics and Chemistry of Wave Packets (Wiley, New York, 2000).

[20] M. G. Moore, O. Zobay, and P. Meystre, Phys. Rev. A 60, 1491 (1999).

[21] H. Stapelfeldt and T. Seideman, Rev. Mod. Phys. 75, 543 (2003).

[22] H. J. Loesch, Annu. Rev. Phys. Chem. 46, 555 (1995).

[23] T. Seideman, J. Chem. Phys. 115, 5965 (2001).

[24] G. Herzberg, Molecular Spectra and Molecular Structure, I Diatomic Molecules (Prentice-Hall, New York, 1939).

[25] P. D. Drummond, K. V. Kheruntsyan, D. J. Heinzen, and R. H. Wynar, Phys. Rev. A 65, 063619 (2002).

[26] M. Mackie, Phys. Rev. A 66, 043613 (2002).

[27] O. Dutta, M. Jääskeläinen, and P. Meystre (unpublished). 